\title{
Doença de Chagas: notificação de triatomíneos no Estado de São Paulo na década de 1990
}

\author{
Chagas' disease: triatomines notification of the \\ São Paulo State during the 1990s
}

\author{
Rubens Antonio da Silva ${ }^{1}$, Dalva Marli Valério Wanderley ${ }^{1}$, Maria de Fátima Domingos ${ }^{1}$, \\ Sueli Yasumaro ${ }^{1}$, Sirle Abdo Salloun Scandar ${ }^{1}$, Clóvis Pauliquévis-Júnior ${ }^{1}$, \\ Susy Mary Perpétuo Sampaio ${ }^{1}$, Luiz Takakuํㅜ e Vera Lúcia Cortiço Corrêa Rodrigues ${ }^{1}$
}

\begin{abstract}
RESUM0
O objetivo deste estudo foi analisar a vigilância da doença de Chagas no Estado de São Paulo, através da notificação, na década de 1990. As informações foram originadas quando da notificação de triatomíneos pelos moradores e seguiram o normatizado pelo Programa de Controle. Foram recebidas 20.563 notificações de triatomíneos com queda ao longo dos anos, sendo esta mais acentuada na região que compreende a área de maior freqüência de encontro de Panstrongylus megistus. Cada notificação correspondeu em média a 1,3 exemplares de triatomíneos capturados (mediana=1), predominantemente no intradomicílio, enquanto nos atendimentos a média de insetos coletados foi de 3,6 (mediana=2), presentes na maioria no peridomicílio. A distribuição das notificações permitiu demarcar três áreas distintas do Estado: área 1- compreendida pelas regiões de São José do Rio Preto, Araçatuba e parte de Presidente Prudente; área 2- São Vicente e Sorocaba; área 3- municípios situados a nordeste da região de Campinas. A análise mostrou que a vigilância entomológica através da notificação de triatomíneos, a despeito da queda das mesmas, não tem detectado colônias intradomiciliares associadas a infecção por Trypanosoma cruzi que possam dar origem à transmissão vetorial da doença de Chagas humana.
\end{abstract}

Palavras-chaves: Doença de Chagas. Programa de Controle. Notificações de triatomíneos. Vigilância entomológica.

\begin{abstract}
The objective of this study was to analyze the surveillance of Chagas' disease in São Paulo State through notifications registered from 1990 to 1999. The information originated when notification of triatomines was announced by inhabitants and the established control program was followed. 20,563 triatomine notifications were received, showing a decrease over the years, with more accentuated decreases in the area of greatest frequency of Panstrongylus megistus. Each notification corresponded to an average of 1.3 examples of captured triatomines ( median $=1)$, predominantly in the intradomicile area, while during attendance, the mean number of collected insects was 3.6 ( median $=2$ ), mostly present in the peridomicile area. The notification distribution permitted the demarcation of three different areas in the state: area 1, comprising the areas of São José do Rio Preto, Araçatuba and part of Presidente Prudente; area 2, São Vicente and Sorocaba; area 3, municipalities located to the northeast of the Campinas region. Analysis showed that entomological surveillance through triatomine notification, despite the fall in the same, has not detected intradomiciliary colonies associated with Trypanosoma cruzi which could give rise to vectorial transmission of Chagas' disease.
\end{abstract}

Key-words: Chagas' disease. Control Program. Triatomine notification. Entomological surveillance.

\footnotetext{
1. Superintendência de Controle de Endemias, São Paulo, SP.

Endereço para correspondência: Dr. Rubens Antonio da Silva. SUCEN. Rua Paula Souza 166/5ํo andar, Luz, 01027-000 São Paulo, SP.

Telefax: 5511 3227-0622

e-mail: rubens@ sucen.sp.gov.br

Recebido em 07/10/2005

Aceito em 10/8/2006
} 
A possibilidade da notificação de focos de triatomíneos, utilizando elementos da própria comunidade, proposta inicialmente por Dias, em $1957^{3}$ e Freitas ${ }^{11}$, em 1963, foi implementada por Silva em $1970^{21}$ e conduzido pela Superintendência de Controle de Endemias (SUCEN). A experiência de um ano em área piloto apresentou resultados muito favoráveis, mas a proposta não se estendeu para o conjunto do Estado com exceção do Vale do Ribeira, no início da década de 1980, conforme descrito por Patucci em 1989 ${ }^{15}$. № âmbito do controle, a participação da população na notificação de insetos suspeitos de serem triatomíneos passou a ser incentivada, no Estado como um todo, a partir de 1983. Esse incentivo consistiu na realização de pesquisa domiciliar em resposta a cada notificação de triatomíneo encaminhada por qualquer munícipe, numa perspectiva de se alcançar maior envolvimento da população na detecção de colônias de triatomíneos em seus domicílios. Desde então, a norma técnica em vigor preconizou, além das pesquisas rotineiras de triatomíneos, 0 atendimento à notificação em prazo não superior a 30 dias, levando-se em consideração que um pronto atendimento permitiria maior envolvimento da população na detecção de colônias de triatomíneos. Ao mesmo tempo, houve um investimento em recursos humanos da área de educação em saúde no serviço de controle, passando esses profissionais a atuar em conjunto com as equipes de campo, visando incrementar 0 componente educativo do programa junto aos moradores, por meio do repasse de informações necessárias à compreensão da importância do trabalho de pesquisa de triatomíneos, orientando-os quanto aos locais mais habituais de encontro dos mesmos ${ }^{25}$.

Com a reformulação do Programa de Controle da Doença de Chagas ( PCDCh) no Estado de São Paulo, realizada no ano de 1989, as atividades de pesquisas rotineiras de triatomíneos passaram a ser direcionadas apenas para as áreas infestadas e foi priorizada a atividade de notificação pelos moradores como medida para a descoberta de colônias de triatomíneos ${ }^{19}$. 0 encaminhamento de insetos suspeitos de serem triatomíneos por intermédio das Unidades Básicas de Saúde, das Escolas ou diretamente aos Serviços Regionais da SUCEN, desencadeia uma pesquisa na casa notificante, sempre que 0 inseto identificado tratar-se de triatomíneo. Avaliações realizadas demonstraram maior eficácia dessa atividade, medida pelos percentuais de casas positivas entre as pesquisadas em atendimento a notificação quando comparados com as pesquisas programadas em rotina e a maior possibilidade de detectar insetos dentro das unidades domiciliares que a atividade de pesquisa integral em uma localidade ${ }^{2324}$.

No Estado de São Paulo, as espécies de triatomíneos mais freqüentemente coletadas nas pesquisas realizadas pelas equipes de campo são: Triatoma sordida, encontrados em maior concentração na área do planalto, incluindo as regiões de Ribeirão Preto, São José do Rio Preto e Araçatuba, Rhodnius neglectus que coincide com a área de distribuição do T. sordida; Panstrongylus megistus nas regiões do Vale do Ribeira, Sorocaba, Campinas e em municípios da região de Ribeirão Preto, que fazem divisa com o Estado de Minas Gerais e Triatoma tibiamaculata disperso na região sul, principalmente no Vale do Ribeira ${ }^{25}$. Estas espécies têm hábitos peridomiciliares e invadem esporadicamente 0 interior das residências.

0 objetivo deste estudo foi analisar a vigilância da doença de Chagas por meio da notificação de triatomíneos no Estado de São Paulo, na década de 1990, com vistas a implementar 0 funcionamento adequado desse sistema de vigilância.

\section{MATERIAL E MÉTODOS}

Os dados foram originados a partir da notificação de triatomíneos realizada pela população aos Serviços Regionais da SUCEN. Os trabalhos seguem as normas no PCDCh desenvolvido no Estado de São Paulo para o período de 1990 a $1999^{19}$. Quando o morador notifica um triatomíneo, o Serviço programa uma visita a moradia correspondente, pesquisando-a integralmente com direcionamento para os locais de abrigo de animais que são utilizados como fonte alimentar pelos triatomíneos, numa atividade designada atendimento à notificação.

Durante 0 trabalho de pesquisa no campo, as informações foram transcritas em boletim padronizado para todo 0 Estado, sendo selecionadas para esta avaliação as variáveis município, data de captura, atividade realizada (notificação ou atendimento a notificação) , local de captura (intradomicílio ou peridomicílio), espécie, estádio evolutivo e infecção por Trypanosoma cruzi. Estas informações foram codificadas e digitadas em programa informatizado e as freqüências extraídas através de programas de análise.

Os 645 municípios do Estado de São Paulo foram distribuídos em 10 Serviços Regionais da Superintendência do Controle de Endemias, aqui denominadas regiões (Figura 1).

\section{RESULTAD0S}

Na década de 1990, foram recebidas no Estado de São Paulo 20.563 notificações de triatomíneos, procedentes de 430 municípios, sendo todas atendidas. Houve concentração de notificações em três áreas distintas, quais sejam: - área 1, constituída pelas regiões de São José do Rio Preto, Araçatuba e parte de Presidente Prudente; - área 2, regiões de São Vicente e Sorocaba; e - área 3, municípios situados a nordeste da região de Campinas. Constatou-se que 215 (33,3\%) municípios dos existentes não apresentaram notificação de triatomíneo e que 61 (9,4\% dos existentes) encaminharam mais do que 100 notificações no período ( Figura 2).

A comparação do número de notificações recebidas, referentes aos primeiros 5 anos da década com os 5 últimos, apontou queda de $36,1 \%$. Esta queda foi mais acentuada nas regiões de São Vicente, Ribeirão Preto, Campinas e Sorocaba, cujos percentuais variaram, em ordem crescente, de 48,2 a 61. Para a região de Presidente Prudente, a queda observada foi a menor do estado com 3,5\% (Tabela 1). No mesmo período, houve queda $(-7,3 \%)$ na população rural do Estado, sendo a mesma presente na maior parte das regiões. 


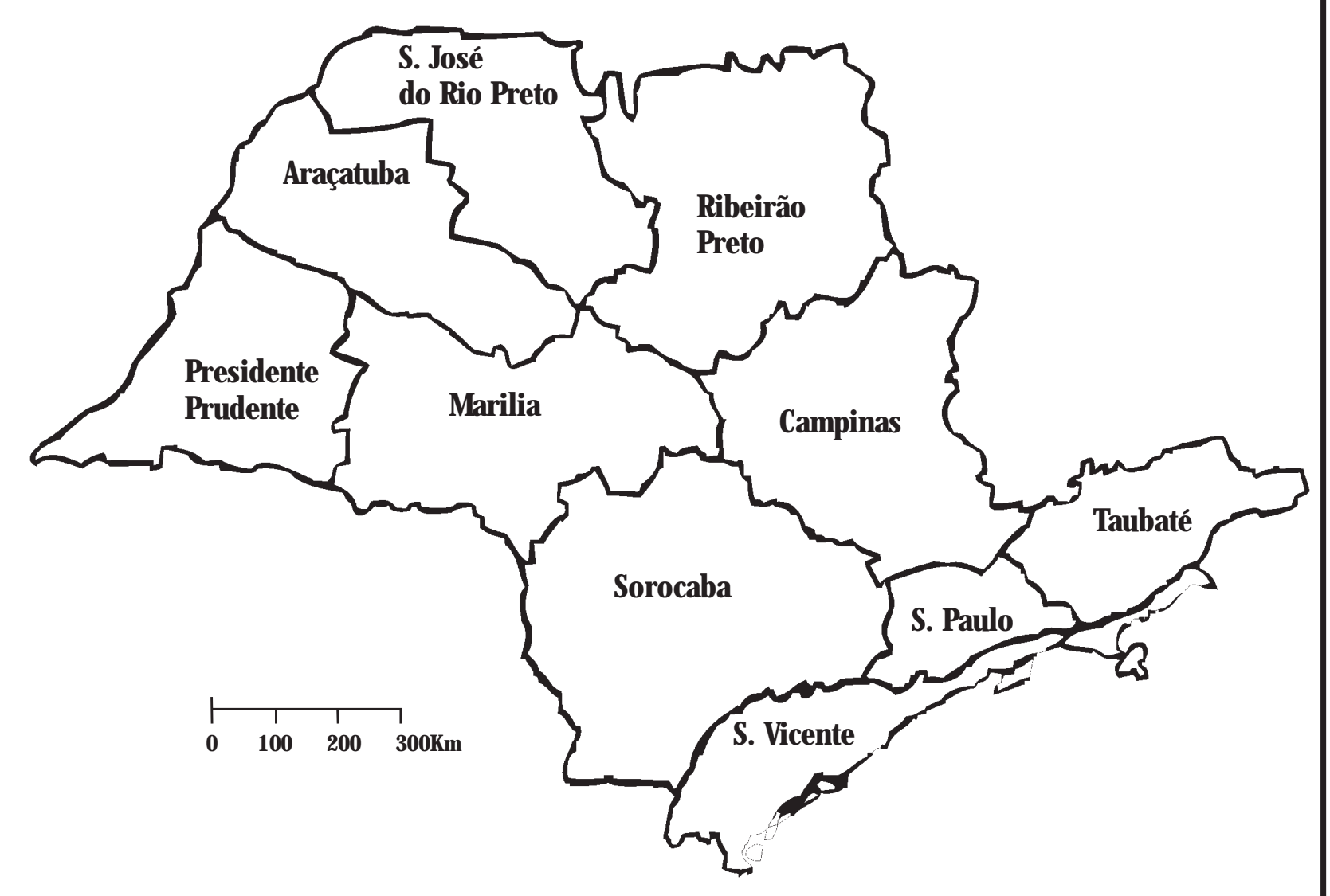

Figura 1 - Abragência dos serviços regionais da Superintendência do Controle de Endemias. Estado de São Paulo.

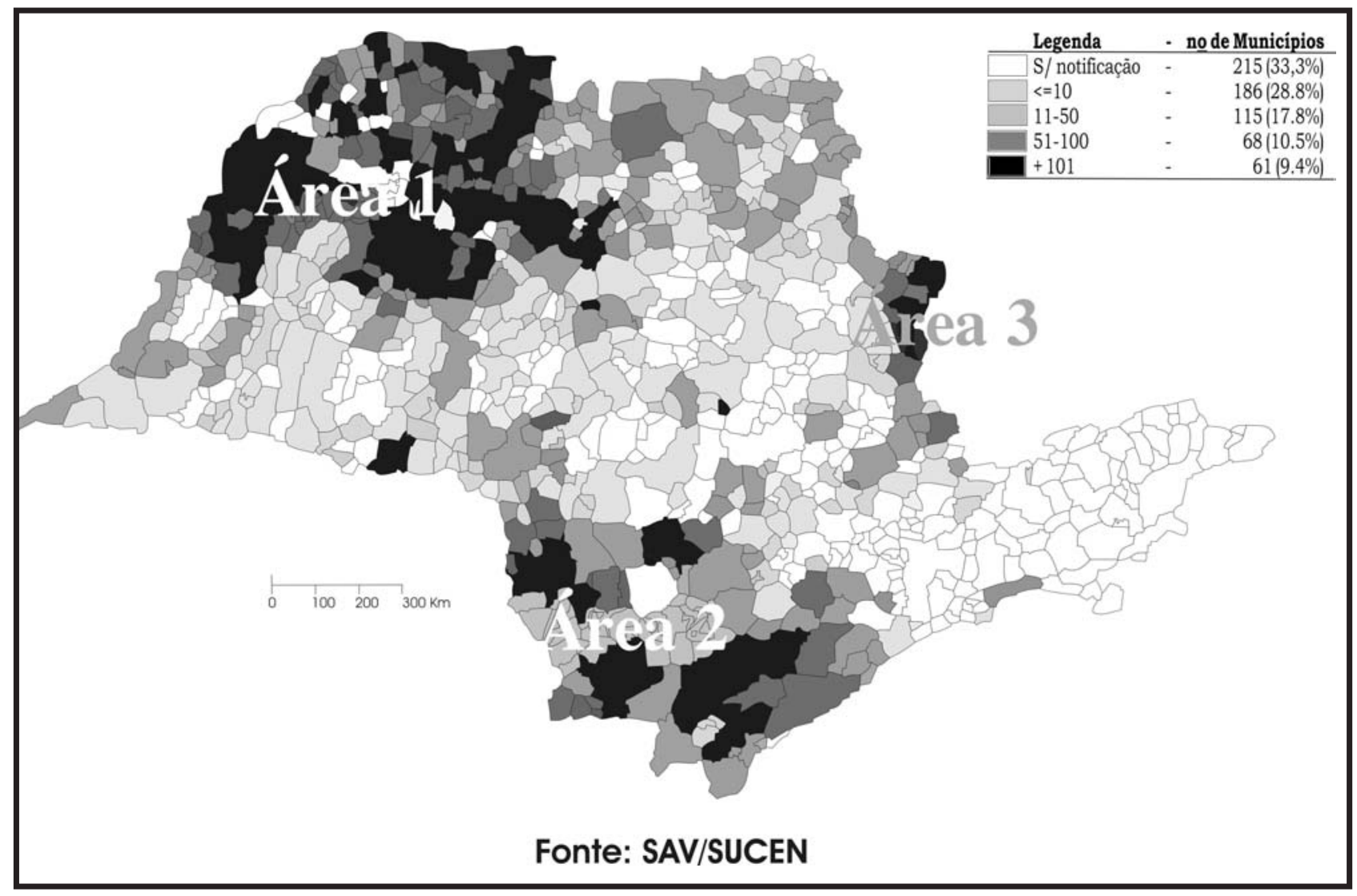

Figura 2 - Distribuição das notificações recebidas segundo município. Estado de São Paulo, 1990 a 1999. 
Tabela 1 - Comparação entre o número de notificações recebidas e população rural nos 5 anos inicias da década de 1990 e os 5 anos finais segundo regióes, Estado de São Paulo.

\begin{tabular}{lrrrrrr}
\hline & \multicolumn{3}{c}{ Notificações - Período } & & \multicolumn{2}{c}{ Variação - Período \% } \\
\cline { 2 - 3 } \cline { 7 - 7 } Região & 1990-1994 & $1995-1999$ & total & & $\begin{array}{c}\text { notificações } \\
\text { rural* }\end{array}$ & população \\
São Vicente & 836 & 433 & 1.269 & & $-48,2$ & 1,9 \\
Sorocaba & 1.895 & 739 & 2.634 & & $-61,0$ & 0,1 \\
Campinas & 900 & 360 & 1.260 & & $-60,0$ & $-6,3$ \\
Ribeirão Preto & 579 & 260 & 839 & & $-55,1$ & $-14,4$ \\
São José Rio Preto & 3.807 & 2.735 & 6.542 & & $-28,1$ & $-12,3$ \\
Araçatuba & 3.484 & 2.520 & 6.004 & & $-27,6$ & $-14,1$ \\
Presidente Prudente & 711 & 686 & 1.397 & & $-3,5$ & $-8,1$ \\
Maŕlia & 338 & 280 & 618 & & $-17,1$ & $-16,2$ \\
\hline Total & 12.550 & 8.013 & 20.563 & & $-36,1$ & $-7,3$ \\
\hline
\end{tabular}

Fonte: SAV/SUCEN/*IBGE

Os atendimentos às notificações resultaram positivos em $26,3 \%$, ou seja, em 5.411 domicílios, novos exemplares de triatomíneos foram encontrados nas pesquisas realizadas pelas equipes de campo (Tabela 2). As regiões de São José do Rio Preto e Araçatuba foram os que receberam maior (61\%) número de notificações e responderam por $65,7 \%$ dos triatomíneos coletados nos atendimentos. Maior percentual de positividade nos atendimentos à notificação foi verificado na região de Ribeirão Preto com 52,4. Os moradores foram responsáveis pelo encaminhamento de
27.647 exemplares de triatomíneos, na atividade de notificação, sendo que a maioria pertencia à fase adulta $(82,7 \%)$ e foram coletados no intradomicílio (88,3\%). Nos atendimentos a estas notificações, as equipes de campo coletaram mais 19.537 exemplares, sendo que 75,6\% destes estavam no peridomicílio e tratavam-se de ninfas (60,9\%). Verificou-se que cada morador notificou, em média, 1,3 exemplares de triatomíneos ( mediana $=1$ ) e nos atendimentos a estas notificações 3,6 exemplares (mediana=2) foram coletados, em média. Embora a região de São Vicente tenha apresentado o menor número de unidades domiciliares positivas nos atendimentos (apenas 7), esta região ao lado da de Campinas, apresentaram as maiores densidades de triatomíneos, com valores médios de 10,4 e 12,3 exemplares, respectivamente. No entanto, os valores das medianas apontaram distribuição heterogênea das colônias, quando se considera 0 número de exemplares coletados nas casas.

Do total de 47.184 triatomíneos capturados destacam as espécies T. sordida e P. megistus com 33.741 (71,5\%) e 9.625 (20,4\%) exemplares coletados, respectivamente (Tabela 3). As notificações ocorreram em todos os meses do ano, sendo observados para área de T. sordida maior número de recebimento nos meses de abril, maio e junho, enquanto para área de P. megistus se concentraram nos meses de outubro e novembro. Os maiores índices de infecção natural por T. cruzi foram observados para as espécies $P$. megistus ( $5,3 \%$ na

Tabela 2 - Notificações recebidas e positivas e triatomíneos coletados pelos moradores e equipes de campo segundo regiões. Estado de São Paulo, 1990 a 1999.

\begin{tabular}{|c|c|c|c|c|c|c|c|c|c|}
\hline \multirow[b]{2}{*}{ Região } & \multirow{2}{*}{$\begin{array}{c}\text { Notificações } \\
\text { recebidas }\end{array}$} & \multicolumn{2}{|c|}{ Atendimentos } & \multicolumn{2}{|c|}{ Triatomíneos coletados } & \multicolumn{2}{|c|}{ Média } & \multicolumn{2}{|c|}{ Mediana } \\
\hline & & positivos & $\%$ & $\begin{array}{l}\text { p/ moradores } \\
\text { (notificação) }\end{array}$ & $\begin{array}{l}\text { Pelas equipes de campo } \\
\text { (atend notif) }\end{array}$ & (1) & $(2)$ & (1) & $(2)$ \\
\hline São Vicente & 1.269 & 7 & 0,5 & 1.408 & 73 & 1,1 & 10,4 & 1,0 & 3,0 \\
\hline Sorocaba & 2.634 & 85 & 3,2 & 3.195 & 198 & 1,2 & 2,3 & 1,0 & 1,0 \\
\hline Campinas & 1.260 & 228 & 18,1 & 1.870 & 2.826 & 1,4 & 12,3 & 1,0 & 3,0 \\
\hline Ribeirão Preto & 839 & 440 & 52,4 & 1.272 & 1.546 & 1,5 & 3,5 & 1,0 & 2,0 \\
\hline São José do Rio Preto & 6.542 & 2.139 & 32,7 & 8.703 & 4.784 & 1,3 & 2,2 & 1,0 & 1,0 \\
\hline Araçatuba & 6.004 & 1.847 & 30,8 & 8.519 & 7.997 & 1,4 & 4,3 & 1,0 & 2,0 \\
\hline Presidente Prudente & 1.397 & 486 & 34,8 & 1.913 & 1.401 & 1,3 & 2,8 & 1,0 & 2,0 \\
\hline Marília & 618 & 179 & 28,9 & 767 & 712 & 1,2 & 3,9 & 1,0 & 1,0 \\
\hline Total & 20.563 & 5.411 & 26,3 & 27.647 & 19.537 & 1,3 & 3,6 & 1,0 & 2,0 \\
\hline
\end{tabular}

Fonte: SAV/SUCEN (1) = Triatomíneos coletados $\mathrm{p} /$ moradores $(2)=$ Triatomíneos coletados pelas equipes campo

Tabela 3 - Infecção natural dos triatomíneos coletados segundo espécie e atividade. Estado de São Paulo, 1990 a 1999.

\begin{tabular}{|c|c|c|c|c|c|c|c|c|c|c|c|c|}
\hline \multirow[b]{3}{*}{ Espécies } & \multicolumn{6}{|c|}{ Notificação } & \multicolumn{6}{|c|}{ Atendimento à notificação } \\
\hline & \multicolumn{2}{|c|}{ adultos } & \multicolumn{2}{|c|}{ ninfas } & \multicolumn{2}{|c|}{ total } & \multicolumn{2}{|c|}{ adultos } & \multicolumn{2}{|c|}{ ninfas } & \multicolumn{2}{|c|}{ total } \\
\hline & colet & \%* & colet & $\% *$ & colet & $\% *$ & colet & $\% *$ & colet & $\% *$ & colet & $\% *$ \\
\hline T. infestans & 3 & 0,0 & 0 & 0,0 & 3 & 0,0 & 21 & 0,0 & 87 & 0,0 & 108 & 0,0 \\
\hline P. megistus & 5.578 & 4,9 & 544 & 10,5 & 6.122 & 5,3 & 2.229 & 17,2 & 1.274 & 7,9 & 3.503 & 16,7 \\
\hline T. sordida & 14.058 & 0,3 & 4.043 & 0,9 & 18.101 & 0,4 & 5.175 & 0,8 & 10.465 & 0,3 & 15.640 & 0,5 \\
\hline R. neglectus & 2.526 & 0,4 & 110 & 0,0 & 2.636 & 0,4 & 164 & 0,0 & 103 & 0,0 & 267 & 0,0 \\
\hline T.tibiamaculata & 627 & 20,5 & 0 & 0,0 & 627 & 20,5 & 0 & 0,0 & 0 & 0,0 & 0 & 0,0 \\
\hline Outros & 154 & 2,4 & 0 & 0,0 & 154 & 2,4 & 5 & 0,0 & 14 & 0,0 & 19 & 0,0 \\
\hline Total & 22.946 & 2,2 & 4.697 & 2,2 & 27.647 & 2,3 & 7.594 & 5,9 & 11.943 & 1,9 & 19.537 & 3,0 \\
\hline
\end{tabular}

Fonte: SAV/SUCEN *\% infecção natural 
notificação e 16,7\% no atendimento) e T. tibiamaculata (20,5\% na notificação). Para P. megistus, destaca-se 0 percentual de 10,5\% de infecção natural obtido para ninfas na atividade de notificação. Porém, é importante destacar que $74,5 \%$ destas ninfas infectadas foram capturadas no peridomicílio. A atividade de notificação foi responsável pela maior captura de exemplares adultos quando comparado com a atividade de atendimento as notificações, responsável pela captura de maior número de ninfas. Não se observou tendência quanto ao estádio evolutivo, local de captura e positividade para T. cruzi para as espécies.

No período do estudo foram notificados 3 exemplares de T. infestans, todos com pesquisa negativa para T. cruzi. Um exemplar, oriundo do município de Tapiratiba, no ano de 1990, foi notificado em residência cujo morador se deslocava regularmente para Minas Gerais, em área com persistência desse vetor, possivelmente sendo o mesmo transportado passivamente entre os seus pertences: outro exemplar foi procedente do município de Sumaré, também introduzido passivamente no estado e 0 terceiro notificado do município de Paulínia. 0 atendimento dos dois primeiros casos resultou em pesquisa negativa. Porém, no terceiro foi localizado um foco com 108 exemplares localizados em ninhos de pássaros, utilizando-se do sangue desses como fonte de alimento. Destaque-se que os 3 municípios pertencem a região de Campinas e que 0 seguimento nos dois primeiros focos resultaram negativos. Para 0 município de Paulínia a revisão da pesquisa e controle realizado, 30 dias após os trabalhos, resultou no encontro de 2 ninfas de 50 estádio desta espécie. Dada a importância deste episódio aos 6 meses do controle inicial, nova pesquisa foi realizada, resultando negativa ${ }^{12}$.

\section{DISCUSSÃ0}

As atividades de vigilância dos vetores da doença de Chagas no Estado de São Paulo têm como objetivo geral manter interrompida a sua transmissão vetorial, atingida na década de 1970, quando os indicadores entomológicos apontavam redução significativa nas capturas de Triatoma infestans, com ausência de infecção por T. cruzi a partir de 1979 e os indicadores sorológicos demonstravam que os índices de infecção em crianças mostravam-se descendentes, tornando-se nulos a partir de $1982^{2022} 25$.

As notificações de triatomíneos, encaminhadas por moradores no período de 1990 a 1999, estiveram concentradas em três áreas distintas do estado. Na primeira, formada pelas regiões de São José do Rio Preto, Araçatuba e parte de municípios que compõem a região de Presidente Prudente, observa-se formação vegetal predominante do tipo cerrado com presença da espécie T. sordida, adaptada ao ambiente peridomiciliar associada a ninho de aves ${ }^{7}$. A segunda é formada pelas regiões de São Vicente e Sorocaba, localizada na área de abrangência da Serra do Mar, com presença de mata atlântica e de espécies como $P$. megistus e T. tibiamaculata. Na terceira, os municípios de Caconde, Divinolândia, São João da Boa Vista, São José do Rio Pardo,
São Sebastião da Grama e Vargem Grande do Sul, pertencentes à região de Campinas, foram os que se destacaram com P. megistus como espécie predominante em colônias mais numerosas do que nas outras áreas (Figura 2). Nesta região, o processo de povoamento e de utilização do solo implicou em destruição considerável da vegetação primitiva, dando lugar à formação de campos. 0 que restou reduz-se, na atualidade, a manchas de matas residuais onde se notam elementos representativos da cobertura vegetal primitiva, entremeados de matas de segunda formação, com concentração de animais vertebrados (marsupiais e roedores) ${ }^{9}$. A partir dessas áreas, este vetor tenta invadir e instalar-se no ambiente humano ${ }^{810}$.

Na região de Presidente Prudente, que apresentou na década de 1990 a menor queda no número de notificações recebidas, um trabalho de incentivo às notificações foi realizado pelas equipes de visitadores sanitários da região junto às escolas e moradias da zona rural com visitas e entrega de material educativo com orientações sobre a importância da notificação (Tabela 1).

Os resultados aqui apresentados permitem afirmar que as espécies de triatomíneos presentes atualmente no estado estão adaptadas ao peridomicílio e que esporadicamente invadem as casas, as quais não encontram condições apropriadas para a formação de colônias, uma vez que não passam despercebidas pelo morador que as coletam e notificam. Por outro lado, a manutenção da presença de colônias peridomiciliares é facilitada pelo desinteresse na limpeza dos arredores da casa para evitar a infestação ${ }^{26}$. Acresce-se que neste ambiente a ação de inseticidas é menos eficaz, embora alguns piretróides sintéticos apresentem ação residual considerável ${ }^{13141617}$. Porém, independentemente do poder residual do inseticida deve-se levar em consideração a renovação do ambiente peridomiciliar ${ }^{4}$.

De acordo com Forattini ${ }^{7}$, a domiciliação do vetor está relacionada a maior ou menor preservação de seus ambientes naturais, do tipo de habitação na área e da possibilidade de abrigo para os triatomíneos, da oferta alimentar existente bem como dos diferentes graus de antropofilia de cada uma das espécies. No entanto, para a maioria dos triatomíneos, a transmissão intradomiciliar do Trypanosoma cruzi está associada à capacidade da espécie promover neste ambiente colônia com muitos indivíduos, que colonizam as casas de maneira permanente e com marcada antropofilia ${ }^{6}$. A ausência de colonização em grau significativo de densidade no ecótopo humano, dissociado de infecção natural, é fator impeditivo para a transmissão da doença humana ${ }^{5}$. Pelos dados aqui apresentados, depreende-se que 0 risco de ocorrência de transmissão é bastante remoto, ou inexistente, uma vez que as colônias com exemplares jovens ( ninfas) de triatomíneos associadas a infecção por T. cruzi foram localizadas fora do ambiente intradomiciliar. Vale ressaltar, que para 0 decênio 1990 a 1999, a sorologia aplicada em moradores de unidades domiciliares onde tenha se dado a captura de triatomíneos infectados por T. cruzi, revelou a presença de sororreagentes com idades compatíveis com a época considerada como limite para a aquisição da doença de Chagas autóctone ${ }^{2}$. 
0 modelo de vigilância desenvolvido no estado permite garantir sustentabilidade às ações, assegurando a detecção precoce dos triatomíneos e sua eliminação, em área de transmissão interrompida, uma vez que vem sendo realizada de forma contínua, com o envolvimento da população e dos serviços locais de saúde e educação, permitindo monitorar a situação. Porém, as ações de educação em saúde tem sido consideradas como fundamentais para manutenção desta sustentabilidade, uma vez que as intervenções verticais têm se mostrado menos eficaz do que as notificações de insetos pela populaçãa ${ }^{23} 25$.

No período em análise, as notificações de triatomíneos apresentaram queda ano a ano. Nesta década, novas problemáticas epidemiológicas que foram surgindo, como a dengue, desde o seu início, e a leishmaniose visceral americana, a partir de 1998, exigiram por parte do serviço maior envolvimento dos profissionais da área de educação em saúde, interferindo nos trabalhos de estímulo às notificações de triatomíneos nas populações. A necessidade de implementar 0 componente educativo para estas doenças dificultou 0 direcionamento das atividades de vigilância de triatomíneos. Salienta-se que se observou queda na população rural, porém acreditamos que a mesma não pode ser atribuída a observada nas notificações, uma vez que em regiões, onde se verificou quedas no número de notificações houve aumento na população rural (Tabela 1).

As ações de Educação em Saúde devem nortear os princípios do Sistema Único de Saúde (SUS) de universalização, descentralização, integralidade e participação da comunidade. Nessa lógica, programas que buscam uma atuação em vigilância à saúde e uma concepção de saúde centrada na promoção da qualidade de vida, como o Programa de Agente Comunitário da Saúde e Programa de Saúde da Família, são modelos de assistência que podem fortalecer esse processo, pois pressupõem a reorganização da atenção básica. Quando estes se estendem à área rural, se tornam um pólo catalisador para a sensibilização dos residentes da área rural, para vigilância e controle de triatomíneos ${ }^{42}$, principalmente, em áreas como no Estado de São Paulo, onde, a partir de 1998, a reestruturação das Unidades Escolares Rurais, no processo de municipalização da rede de ensino, propiciou um esvaziamento desse mecanismo que envolve alunos e professores de escolas rurais, e que foi um dos mais utilizados pelo serviço de controle no estimulo a participação da população. Esse modelo tem sido implantado e implementado no estado, nas diferentes regiões, e será futuramente analisado.

Concordamos com Briceño-León ${ }^{1}$, ao afirmar que a participação popular tem papel cada vez mais importante no contexto das endemias, uma vez que as mesmas são fenômenos coletivos que afetam grande parte da população e, portanto, qualquer política de controle requer a cooperação das pessoas submetidas ao risco. Nesse aspecto, a sustentabilidade da participação da população na vigilância de triatomíneos perpassa também pela reorientação das ações educativas. Ver e manusear instrumentos para entender, no concreto, o ciclo de vida dos vetores, e a doença, por exemplo, podem ser uma forma pedagógica de criar oportunidade para a população participar no processo de conhecimento e prática. Um programa dialógico e participativo em saúde implica que todos atuem por igual, porém com regras diferenciadas num contexto de perspectivas e prioridades tão legítimas e válidas para o Serviço de controle como para a comunidade.

No campo da investigação, destaca-se como importante 0 desenvolvimento de estudos de caráter social que objetivem identificar ações sustentáveis de informação, educação e capacitação dirigidas à população nas diversas faixas etárias, que apoiem as ações do programa através da mudança de práticas no uso e manutenção das habitações, além de investigações que selecionem medidas que estimulem a população a realizar a vigilância entomológica e reorganização do meio no domicílio como um todo, visando implementar a vigilância e diminuir a possibilidade de recolonização de casa pelo vetor ${ }^{18}$.

\section{REFERÊNCIAS BIBLIOGRÁFICAS}

1. Briceño-León R. El contexto político de la participación comunitaria en América Latina. Cadernos de Saúde Pública 14:141-147, 1998.

2. Carvalho ME, Silva RA, Rodrigues VLCC, Oliveira CD. Programa de Controle da Doença de Chagas no Estado de São Paulo: sorologia de moradores como parte de investigação de unidades domiciliares com presença de triatomíneos vetores na década de 1990. Cadernos de Saúde Pública 18:1695-1703, 2002.

3. Dias E. Sugestões preliminares para um plano de erradicação dos transmissores domiciliários da doença de Chagas no Estado de São Paulo. Revista Médica Sul de Minas 2:104-113, 1957.

4. Dias JCP. Problemas e Possibilidades de Participação Comunitária no Controle das Grandes Endemias no Brasil. Cadernos de Saúde Pública 14:19-37, 1998.

5. Dias JCP. Epidemiologia e controle da doença de chagas: que características podem definir espécies/populações de triatomíneos com maior potencial vetorial? Revista da Sociedade Brasileira de Medicina Tropical 34( supl III) :82-85, 2001.

6. Diotaiuti L. 0 risco da domiciliação de novas espécies de triatomíneos. Revista da Sociedade Brasileira de Medicina Tropical 33( supl II) :31-35, 2000.

7. Forattini OP. Biogeografia, origem e distribuição da domiciliação de triatomíneos no Brasil. Revista de Saúde Pública 14:265-299, 1980.

8. Forattini OP, Ferreira AF, Silva EOR, Rabello EX. Aspectos ecológicos da tripanosomíase americana. VIII - Domiciliação de Panstrongylus megistus e sua presença extradomiciliar. Revista de Saúde Pública 11:73-86, 1977a.

9. Forattini OP, Santos JLF, Ferreira OA, Silva EOR, Rabello EX. Aspectos ecológicos da tripanosomíase americana. XVI - Dispersão e ciclos anuais de colônias de Triatoma sordida e de Panstrongylus megistus espontaneamente desenvolvidos em ecótopos artificiais. Revista de Saúde Pública 13:299-313, 1979.

10. Forattini OP, Silva EOR, Ferreira OA, Rabello EX, Lima AR. Aspectos ecológicos da tripanosomíase americana. XI - Domiciliação de Panstrongylus megistus e potencial enzoótico. Revista de Saúde Pública 11:527-550, 1977b.

11. Freitas JLP. Importância do expurgo seletivo dos domicílios e anexos para a profilaxia da moléstia de Chagas pelo combate aos triatomíneos. Arquivos de Higiene e Saúde Pública 28: 217-272, 1963.

12. Leite OF, Alves MJCP, Souza SSL, Mayo RC, Andrade VR, Souza CE, Rangel 0, Oliveira SS, Lima VLC, Rodrigues VLCC, Carvalho ME, Wanderley DMV. Triatoma infestans em área sob vigilância entomológica para doença de Chagas, Estado de São Paulo, Brasil. Revista da Sociedade Brasileira de Medicina Tropical 34:437-443, 2001. 
13. Oliveira-Filho AM. New alternative the control of triatomines in peridomestic buildings. Revista da Sociedade Brasileira de Medicina Tropical 22:53-57, 1989.

14. Oliveira Filho AM. Differences of susceptibility of five triatomine species to pyrethroid insecticides - implications for Chagas Disease Vector Control. Memórias do Instituto Oswaldo Cruz 94 ( supl I) :425-428,1999.

15. Patucci RMJ, Glasser CM, Domingos MF, Ciaravolo RMC. Vigilância sobre a fauna triatomínica da região do Vale do Ribeira, São Paulo, com a participação da população. In: Anais do XI Congresso Brasileiro de Parasitologia, Rio de Janeiro, p.161, 1989.

16. Rodrigues VLCC, Ishihata GK, Silva EOR, Ferraz-Filho NA. Controle dos triatomíneos através do rociado focal com piretróides sintéticos. In: Resumos do XXXIII do Congresso da Sociedade Brasileira de Medicina Tropical, Belo Horizonte, p. 63, 1997.

17. Rodrigues VLCC, Silva RA, Baitelo D, Silva PR. Efeito do inseticida cipermetrina sobre Panstrongylus megistus (Hemiptera - Reduviidae). Revista da Sociedade Brasileira de Medicina Tropical 35 ( supl III):182183, 2002.

18. Schmunis GA. Necessidades de investigacion aplicada em la enfermedad de Chagas: identification de nuevas pautas de investigation ajustadas a lãs demandas actuales de los programas de control. Revista da Sociedade Brasileira de Medicina Tropical 34( supl III) :37-38, 2001.
19. Secretaria de Estado da Saúde. Relatório final do Grupo de Trabalho do Programa de Controle da Doença de Chagas no Estado de São Paulo, 1989.

20. Silva EOR, Guarita OF, Ishihata GK. Doença de Chagas: atividades de controle dos transmissores no Estado de São Paulo, Brasil. Revista Brasileira de Malariologia 31:99-119, 1979.

21. Silva EOR, Maluf J, Corrêa RR. Doença de Chagas: atividades de vigilância entomológica numa área do Estado de São Paulo, Brasil. Revista de Saúde Pública 4:129-45,1970.

22. Silva EOR, Wanderley DMV, Rodrigues VLCC. Triatoma infestans: importância, controle e eliminacão da espécie no Estado de São Paulo, Brasil. Revista da Sociedade Brasileira de Medicina Tropical 31:73-88, 1998.

23. Silva RA, Bonifácio PR, Wanderley DMV. Doença de Chagas no Estado de São Paulo: Comparação entre pesquisa ativa de triatomíneos em domicílios e notificação de sua presença pela população em área sob vigilância entomológica. Revista da Sociedade Brasileira de Medicina Tropical 32:653-659, 1999.

24. Silveira AC. Situação do controle da transmissão vetorial da doença de Chagas nas América. Cadernos de saúde Pública 16 ( supl 2):35-42, 2000.

25. Wanderley DMV. Perspectivas de Controle da Doença de Chagas no Estado de São Paulo. Faculdade de Saúde Pública da Universidade de São Paulo, 1994.

26. Yasumaro S, Gomes AHA, Araújo OE, Wanderley DMV. Vigilância entomológica da doença de Chagas na Região de Sorocaba, Estado de São Paulo, Brasil. Revista da Sociedade Brasileira de Medicina Tropical 26( supl I) : 79, 1993. 